\title{
A nuanced diagnosis with grave implications: importance of a rare ECG Pattern
}

\section{Background}

De winter syndrome is a rare ECG pattern indicating proximal LAD occlusion and has been reported to be found in 2 percent of patients presenting with acute anterior myocardial infarction. This ECG pattern has been described as a STEMI-equivalent. Due to its rarity, recognition of this finding may be delayed and prevent the necessary prompt reperfusion therapy.

\section{Objective}

The objectives of this report are to identify de Winter syndrome on ECG and distinguish this pattern from other ECG entities that may appear visually similar.

\section{Case report}

A 63-year-old man with a history of hypertension, hyperlipidemia, tobacco use, and hepatitis C status-post treatment presented to the Emergency Department with a two-hour history of sudden-onset stabbing central chest pain with radiation to the back. He experienced

\author{
Volume I4 Issue 4 - 202I
}

\author{
Sneha Limaye, Aisha Siraj \\ Department of Heart \& Vascular Center, Case Western Reserve \\ University, USA
}

\begin{abstract}
Correspondence: Sneha Limaye, Department of Heart \& Vascular Center, Case Western Reserve University, Cleveland, OH 44109, USA, Email slimaye@metrohealth.org
\end{abstract}

Received: June 20, 2021 | Published: August 05, 2021

diaphoresis and nausea during the onset of chest pain. The patient was given aspirin and experienced some relief with the third tablet of nitroglycerin. Chest X-ray showed no acute findings. An ECG was performed (Figure 1) and showed upsloping ST-segment depression at the $\mathrm{J}$ point in the precordial leads with progression into tall, symmetric T waves in these leads and mild ST-segment elevation in lead aVR.

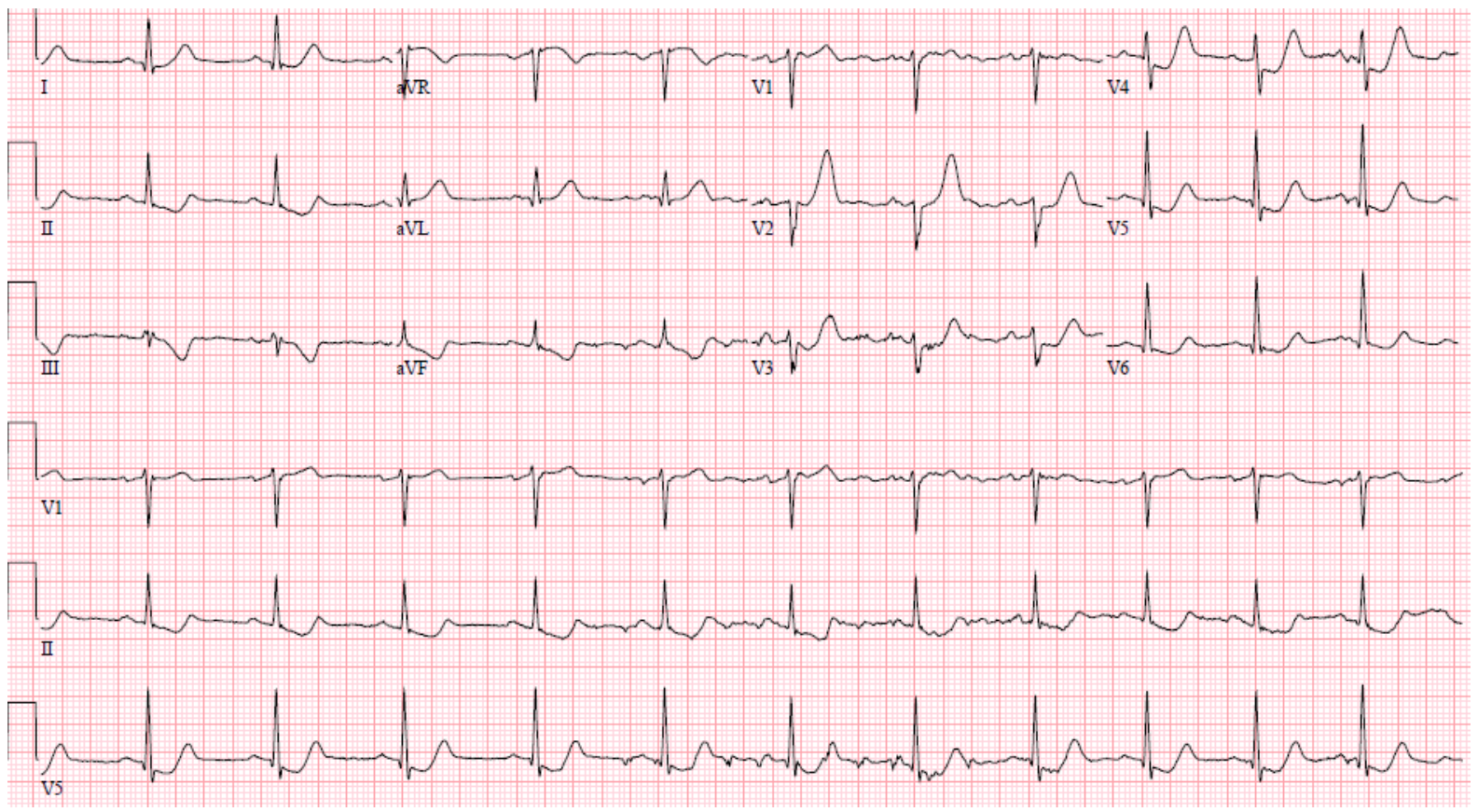

Figure I DeWinter ECG pattern. This ECG shows upsloping ST-segment depression at the J point in the precordial leads with progression into tall, symmetric $\mathrm{T}$ waves in these leads, mild ST-segment elevation in lead aVR, and ST-segment depression in the inferior leads with $\mathrm{T}$ wave inversion in lead III.

The initial troponin value was mildly elevated. The patient was given therapeutic lovenox and ticagrelor. He was taken emergently to the cardiac catheterization laboratory. The cardiac angiogram demonstrated proximal LAD occlusion (Figure 2) and no significant disease of any other coronary artery. Percutaneous coronary intervention (PCI) of the proximal LAD with drug-eluting stent (DES) was performed (Figure 2). After PCI, the patient reported resolution of his symptoms. His post-revascularization ECG showed resolution of the acute ST changes. He was started on dual antiplatelet therapy. A post-revascularization echocardiogram showed focal wall motion abnormalities and a preserved ejection fraction of fifty percent. Guideline-directed medical therapy was started. 


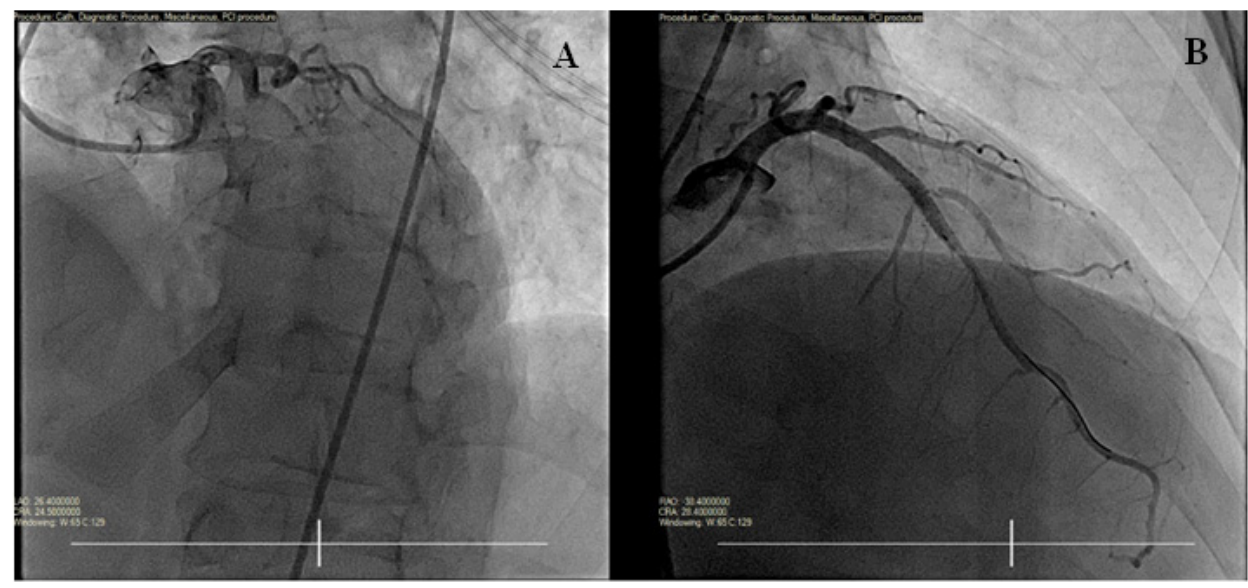

Figure 2 Cardiac catheterization. A: Proximal LAD occlusion. B: Revascularization after stent deployment to the LAD.

\section{Discussion}

This patient was found to have an acutely occluded proximal LAD when taken for emergent cardiac catheterization (Figure 2). The ECG demonstrates de Winter syndrome, a finding specific to proximal LAD occlusion. This syndrome is characterized by upsloping 1-3 millimeter ST-segment depression at the J point in leads V1-V6 which progresses into tall, symmetric $\mathrm{T}$ waves in these leads. ${ }^{1}$ One-to-two millimeter ST-segment elevation in lead aVR can also be seen and was present in this case. Though hyper acute $\mathrm{T}$ waves can be present in hyperkalemia, this electrolyte abnormality is also associated with widened QRS complexes. De Winter syndrome is distinguished from hyperkalemia by the presence of the characteristic upsloping STsegment depressions and absence of widened QRS complexes.

It is important to recognize that patients with ECG findings of de Winter syndrome have a risk of substantial myocardial loss given the association with proximal LAD occlusion, and rapid reperfusion is crucial for this reason. ${ }^{1}$ This ECG finding has been described as a STEMI-equivalent due to its association with large acute myocardial infarction. ${ }^{2}$ Two percent of patients with anterior myocardial infarction were found with de Winter syndrome on ECG. ${ }^{1}$ Clinicians should be highly aware of this characteristic ECG finding to improve triage and outcomes in these patients.

\section{Acknowledgments}

None.

\section{Conflicts of interest}

Author declares there are no conflicts of interest.

\section{Funding}

There was no funding for this report.

\section{References}

1. de Winter RJ, Verouden NJ, Wellens HJ, et al. A new ECG sign of proximal LAD occlusion. N Engl J Med. 2008;359(19):2071-2073.

2. de Winter RW, Adams R, Verouden NJ, et al. Precordial junctional STsegment depression with tall symmetric T-waves signifying proximal LAD occlusion, case reports of STEMI equivalence. $J$ Electrocardiol. 2016;49(1):76-80. 